\title{
T.I.M.S: TaqMan Information Management System, tools to organize data flow in a genotyping laboratory Stéphanie Monnier ${ }^{1}$, David G Cox ${ }^{2}$, Tim Albion ${ }^{3}$ and Federico Canzian*1,4
}

Address: ${ }^{1}$ International Agency for Research on Cancer, F-69372, Lyon, France, ${ }^{2}$ Department of Epidemiology, Harvard School of Public Health, Boston, MA 02115, USA, ${ }^{3}$ Menzies Research Institute, University of Tasmania, Hobart 7001, Australia and ${ }^{4}$ German Cancer Research Center (DKFZ), D-69120, Heidelberg, Germany

Email: Stéphanie Monnier - monnier@iarc.fr; David G Cox - dcox@hsph.harvard.edu; Tim Albion - Tim.Albion@utas.edu.au; Federico Canzian* - f.canzian@dkfz.de

* Corresponding author

Published: 12 October 2005

BMC Bioinformatics 2005, 6:246 doi:10.1 186/147|-2105-6-246
Received: 02 May 2005

Accepted: 12 October 2005

This article is available from: http://www.biomedcentral.com/I47I-2105/6/246

(C) 2005 Monnier et al; licensee BioMed Central Ltd.

This is an Open Access article distributed under the terms of the Creative Commons Attribution License (http://creativecommons.org/licenses/by/2.0), which permits unrestricted use, distribution, and reproduction in any medium, provided the original work is properly cited.

\begin{abstract}
Background: Single Nucleotide Polymorphism (SNP) genotyping is a major activity in biomedical research. The Taqman technology is one of the most commonly used approaches. It produces large amounts of data that are difficult to process by hand. Laboratories not equipped with a Laboratory Information Management System (LIMS) need tools to organize the data flow.
\end{abstract}

Results: We propose a package of Visual Basic programs focused on sample management and on the parsing of input and output TaqMan files. The code is written in Visual Basic, embedded in the Microsoft Office package, and it allows anyone to have access to those tools, without any programming skills and with basic computer requirements.

Conclusion: We have created useful tools focused on management of TaqMan genotyping data, a critical issue in genotyping laboratories whithout a more sophisticated and expensive system, such as a LIMS.

\section{Background}

The completion of the human genome sequence has brought a wealth of data on genetic variation, mostly in the form of single nucleotide polymorphisms (SNPs).

As a consequence, SNP genotyping has recently become a major activity for studies of disease susceptibility and pharmacogenetics. While techniques for ultra-high throughput (hundreds of millions of genotypes scored per year) are becoming available, the vast majority of genotyping laboratories around the world are equipped with technology suited for low- to high-throughput (up to a few million genotypes scored per year). The 5 ' nuclease assay, also known as TaqMan, is one of the main approaches currently used for genotyping $[1,2]$.

Small genotyping laboratories are rarely equipped with sophisticated Laboratory Information Management Systems (LIMS) to follow the flow of information on samples and genotyping results. In a typical workflow (Figure 1 represents the workflow in our laboratory at the Genome Analysis Team, International Agency for Research on Cancer), many files and many different formats are managed. For example, DNA stocks are usually stored in 96-well plates but genotyped in 384-well plates. Sample preparation and handling are often done with different kind of robots (e.g. instruments for DNA extraction, liquid 


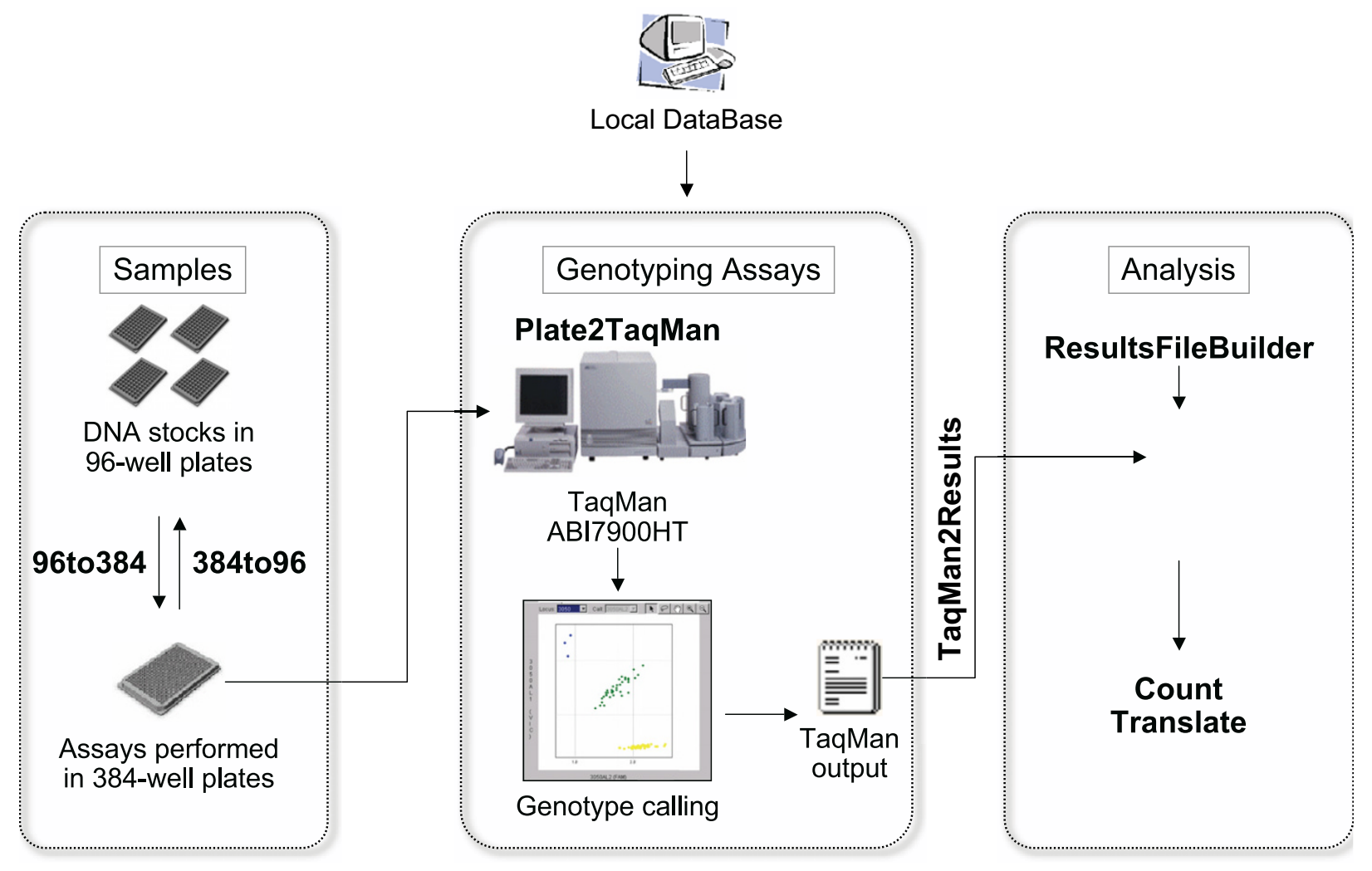

Figure I

Data flow in a SNP genotyping laboratory. Data flow is divided into management of samples, genotyping assays and genotyping results (dotted outlines). In bold, names of macros described in the text. All operations can be interfaced with a local database storing e.g. details about names of samples in plates, sequences of primers and probes used as genotyping assays, genotyping results.

handling robots for set-up of PCR reactions, plate cranes linked with TaqMan instruments), each one with its own input and output file format.

We have developed a software suite for genotyping laboratories, aimed at improving data workflow, saving time and preventing errors linked to managing data by hand. Previously, other groups have developed tool focusing on management of data workflow [3,4]. Differently from previous solutions, we propose a set of independent tools, with a focus on ease of use. Users can download some of the macros or the whole suite, according to their needs. In addition, previously developed programs were tailored to linkage analysis projects, while TIMS is aimed at genotyping in the context of population-based association studies, and therefore it includes several new functions that allow to treat more easily large numbers of sample plates, and to generate input files for downstream software that is not relevant to linkage analysis projects.

\section{Implementation}

TIMS is a suite of tools written in Visual Basic. Given that genotyping data are usually represented in tabular format, we have chosen to use the version of Visual Basic embedded in Microsoft Excel. This offers the advantage of being available to most computer users, and allows interopera- 
bility between Windows and MacOS. The different tools have the format of Visual Basic macros, and they are used at different steps of the workflow (Figure 1):

\section{Sample processing}

We used a simple plate map format to document the localization of DNA samples (one file per plate). Often, both 96-well and 384-well plates are used in a laboratory, which creates the necessity of conversion between the two formats.

Macro "96to384" is used to create the map of a 384-well plate, starting from the map of four 96-well plates. Two ways of interleaving sample location from 96-well to 384well plates are proposed ("Z" or "N", reading from left to right or from top to bottom). The user has to provide a list of files corresponding to 96-well plates to be read by the program and treated in batch. An optional function is offered to the user, to create automatically all the Taqman input files from the newly created 384-well plates. This is the function of the macro "PlateToTaqMan", which transforms the plate maps into files that can be directly used as TaqMan input files, in batch for 96-well plate and 384well plates.

Reciprocally, knowing the composition of a 384-well plate in advance, a user may want to reconstruct the four 96-well DNA source plates. The macro "384to96" gives the structure of the 96-well plates to start with, in order to have the required 384-well plate.

\section{Results management}

The program "ResultsFileBuilder" builds an Excel file, ready to receive the genotyping results. The format of the result file is compatible with our previously published software [5]. The macro copies the list of samples (including information on plate number and location within plates) from a series of plate map files.

The output of the TaqMan instrument is a text file, with genotype information that has to be parsed to make use of it. Another macro, called "TaqMan2Results", is used to transfer the results from the Taqman output file into the results file described above.

Several functions have been built in this macro:

- The possibility to compare analysis of the same assay read by two users. In our laboratory, we routinely perform double blind reading of each TaqMan plate, for quality control. In this case, the macro imports and compares all genotype calls from a batch of plates, read by both users. Any discrepancies are highlighted and the operator is prompted to resolve them manually.
- Transfer of all results in the results file. We have defined a sample as the concatenation of three pieces of information: sample_id, plate name and position of the well in the plate. This has been introduced in order to allow for the existence of samples duplicated on purpose.

- Duplication of a subset of samples, on the same plate or on different plates, is often used for quality control purposes. Knowing which samples have been duplicated for quality control, the macro looks for the results obtained for the first genotype and for the control genotype (identified in the TaqMan input and output files as "Qc_sample_id"), in order to compare them. The macro generates a quality control report file, where it flags all differences between samples and their controls, as well as their position in plates, to make the quality control checking easier.

\section{Analysis}

A macro called "Count" has also been created to make some basic statistical analysis of the results. For each SNP existing in the results file, it counts the frequency of each allele and of each genotype, and checks Hardy-Weinberg equilibrium, using a chi-square test.

Finally, we have added a macro "Translate", which converts the results from the four letters representing nucleotides into numbers. In further analysis, some pieces of software require numbers instead of letters for genotyping data, for instance Haploview [6] and/or TagSNPs $[7,8]$. The macro creates the starting point file for those applications. The user has still to arrange the polymorphisms according to their physical order and keep only the SNPs of interest depending e.g. on their frequencies and the blocks structure in the input file (TagSNPs software).

\section{Conclusion}

We have created a set of user-friendly tools to make laboratory life easier and processing of genotyping data safer and quicker. The use of Microsoft Excel Visual Basic allows access to a wide range of users, working on PC or Mac computers.

This set of programs can be particularly helpful in laboratories where a full-fledged LIMS is not available. They complement (and can in the future be interfaced with) our database, which stores information on polymorphisms under study and their corresponding genotyping assays [9].

The macros we are presenting have been tailored to the instruments we have in our laboratory. However they can easily be modified in order to suit specific requirements, e.g. genotyping chemistries different from the 5 ' nuclease assay that can run on an Applied Biosystems Sequence 
Detection System, such as Amplifluor [10], or MGB Eclipse [11]. To this end, the source code of the macros can be freely consulted (see Additional file 1) and altered by using the Visual Basic Editor embedded in MS Excel.

\section{Availability and requirements Project name}

TIMS, Macroshack.

\section{Project home page}

http://bioinformatics.org/macroshack/prog list.html

\section{Operating system(s)}

The software has been thoroughly tested in Excel 97 and 2000 under various versions of Windows (English versions), Excel 98 of MacOS 9 and Excel X of MacOS X (English versions).

\section{Programming language}

Visual Basic. The source code of all programs is accessible by use of the Visual Basic Editor included in MS Excel.

\section{License}

GNU General Public License.

\section{Any restrictions to use by non-academics?}

None.

\section{Authors' contributions}

FC has mainly conceived the tasks of the macros.

All the authors have contributed to writing the code.

SM and FC have prepared the first draft of the manuscript, and all co-authors contributed to the final draft.

\section{Additional material}

\section{Additional File 1}

Source code of software described in article. Visual Basic source code of macros described in the article. Each macro is composed of several modules or parts of Visual Basic code. Variables are defined by the key word "Dim" and comments are introduced after " ' ". Each piece of code is functional if embedded between "Sub NameOfTheSub" And "End Sub".

Click here for file

[http://www.biomedcentral.com/content/supplementary/14712105-6-246-S1.doc]

\section{Acknowledgements}

Stéphanie Monnier was recipient of a Special Training Award of the International Agency of Research of Cancer. The authors thank the members of the Genome Analysis Team of IARC for extensive testing of the software tools.

\section{References}

I. Morin PA, Saiz R, Monjazeb A: High-throughput single nucleotide polymorphism genotyping by fluorescent 5 ' exonuclease assay. Biotechniques 1999, 27:538-544.

2. Ranade K, Chang MS, Ting CT, Pei D, Hsiao CF, Olivier M, Pesich R, Hebert J, Chen YD, Dzau VJ, Curb D, Olshen R, Risch N, Cox DR, Botstein D: High-throughput genotyping with single nucleotide polymorphisms. Genome Res 200I, I I:1262-1268.

3. Li J-L, Deng H, Dong-Bing L, Fuhua X, Chen J, Gao G, Recker R, Deng H-W: Toward High-Throughput Genotyping: Dynamic and Automatic Software for Manipulating Large-Scale Genotype Data Using Fluorescently Labeled Dinucleotide Markers. Genome Res 200I, I I: 1304-1314.

4. Hampe J, Wollstein A, Lu T, Frevel H-J, Will M, Manaster C, Schreiber $S:$ An Integrated system for high throughput TaqMan ${ }^{\mathrm{TM}}$ based SNP genotyping. Bioinformatics Application Note 200I, 17:654-655.

5. Cox DG, Canzian F: Genotype transposer: automated genotype manipulation for linkage disequilibrium analysis. Bioinformatics 200I, 17:738-739.

6. Barrett JC, Fry B, Maller J, Daly MJ: Haploview: analysis and visualization of LD and haplotype maps. Bioinformatics 2005, 21:263-265.

7. Stram DO, Leigh Pearce C, Bretsky P, Freedman M, Hirchhorn JN, Altshuler D, Kolonel LN, Henderson BE, Thomas DC: Modeling and E-M estimation of haplotype-specific relative risks from genotype data for a case-control study of unrelated individuals. Hum Hered 2003, 55: $179-190$.

8. Stram DO, Haiman CA, Hirschhorn JN, Altshuler D, Kolonel LN, Henderson BE, Pike MC: Choosing haplotype-tagging SNPs based on unphased genotype data using preliminary sample of unrelataed subjects with an example from the Multiethnic Cohort Study. Hum Hered 2003, 55:27-36.

9. Landi S, Gemignani F, Monnier S, Canzian F: A database of single nucleotide polymorphisms and a genotyping microarray for genetic epidemiology of lung cancer. Exp Lung Res 2005, $31: 223-258$.

10. Petkov PM, Ding Y, Cassell MA, Zhang W, Wagner G, Sargent EE, Asquith S, Crew V, Johnson KA, Robinson P, Scott VE, Wiles MV: An efficient SNP system for mouse genome scanning and elucidating strain relationships. Genome Res 2004, 14:|806-|8| I.

II. Afonina IA, Reed MW, Lusby E, Shishkina IG, Belousov YS: Minor groove binder-conjugated DNA probes for quantitative DNA detection by hybridization-triggered fluorescence. Biotechniques 2002, 32:940-949.

Publish with BioMed Central and every scientist can read your work free of charge

"BioMed Central will be the most significant development for disseminating the results of biomedical research in our lifetime. "

Sir Paul Nurse, Cancer Research UK

Your research papers will be:

- available free of charge to the entire biomedical community

- peer reviewed and published immediately upon acceptance

- cited in PubMed and archived on PubMed Central

- yours - you keep the copyright 